\title{
artigo
}

Barbosa, I.S.F.; Mendonça, C.F.S.; Andrade da Silva, L.; Neves, M.P.; Robson da Silva, R.; Alencar, I.F.; Araújo, M.S.;

Produções acadêmicas sobre a assistência destinada às pessoas com problemas relacionados ao abuso de álcool. Um estudo de revisão

\section{Produções acadêmicas sobre a assistência destinada às pessoas com problemas relacionados ao abuso de álcool. Um estudo de revisão}

\author{
Academic productions on assistance for people with problems related to alcohol abuse. A review study \\ Producciones académicas sobre asistencia a personas con problemas relacionados con el abuso de alcohol. Un estudio \\ de revisión
}

\begin{abstract}
RESUMO
O uso nocivo do álcool é um sério problema de saúde publica mundial, afeta diferentes culturas e classes sociais. 0 consumo remota desde a antiguidade, alcoolismo foi ao longo da marcado por diversas formas de exclusão. Objetivo: descrever o estado da arte da atuação de enfermagem junto a pessoas com problemas de uso abusivo de álcool, expresso na literatura científica nacional. Método: trata-se de um estudo de revisão integrativa de literatura, o qual respeitou as seis etapas propostas. Resultado: foram encontrados 25 estudos desenvolvidos por enfermeiros, estes foram agrupados em duas grandes categorias, compreendidas por Atenção básica e sua relação com a saúde mental, e Atenção hospitalar em saúde mental, estas foram subdivididas para ume melhor compreensão dos resultados. Conclusão: Através deste estudo foi possível traçar um panorama das produções acadêmicas de enfermagem, as quais destacassem a atuação do profissional enfermeiro.
\end{abstract}

DESCRITORES: Atenção Primária à Saúde; Saúde Mental; Álcool.

\section{ABSTRACT}

Harmful use of alcohol is a serious public health problem worldwide, affecting different cultures and social classes. Remote consumption since antiquity, alcoholism has been marked throughout by various forms of exclusion. Objective: to describe the state of the art of nursing practice with people with alcohol abuse problems, expressed in the national scientific literature. Method: this is an integrative literature review study, which respected the six steps proposed. Result: 25 studies developed by nurses were found, these were grouped into two major categories, comprised by primary care and its relationship with mental health, and hospital care in mental health, these were subdivided for a better understanding of the results. Conclusion: Through this study it was possible to draw an overview of academic nursing productions, which highlighted the role of the professional nurse. DESCRIPTORS: Primary Health Care; Mental Health; Alcohol.

\section{RESUMEN}

El consumo nocivo de alcohol es un grave problema de salud pública en todo el mundo, que afecta a diferentes culturas y clases sociales. El consumo remoto desde la antigüedad, el alcoholismo se ha caracterizado por diversas formas de exclusión. Objetivo: describir el estado del arte de la práctica de enfermería con personas con problemas de abuso de alcohol, expresado en la literatura científica nacional. Método: se trata de un estudio de revisión integradora de la literatura, que respetó los seis pasos propuestos. Resultado: se encontraron 25 estudios desarrollados por enfermeros, estos se agruparon en dos grandes categorías, comprendidas por la atención primaria y su relación con la salud mental, y la atención hospitalaria en salud mental, estos fueron subdivididos para una mejor comprensión de los resultados. Conclusión: A través de este estudio fue posible trazar un panorama de las producciones académicas de enfermería, que destacó el rol del profesional de enfermería.

DESCRIPTORES: Atención Primaria a la Salud; Salud mental; Alcohol.

RECEBIDO EM: 31/10/2020 APROVADO EM: 19/01/2021

\section{Iolanda de Souza Francisco Barbosa}

Enfermeira. Especialista em Estratégia em Saúde da Família pela Universidade Castelo Branco.

ORCID: 0000-0001-8940-7138 


\section{Conceição de Fátima de Sousa Mendonça}

Enfermeira. Especialista em Saúde pública com ênfase em ESF pela Faculdade FAVENI: Venda Nova do Imigrante. ORCID: 0000-0002-8728-2252

\section{Leandro Andrade da Silva}

Enfermeiro. Pós-Doutor pelo Programa de Pós-graduação em Enfermagem da Universidade do Estado do Rio de Janeiro (PPGEnf/UERJ). Especialista em Saúde Mental e Atenção Psicossocial pela Escola Nacional de Saúde Pública Sérgio Arouca/ENSP da Fundação Oswaldo Cruz (FIOCRUZ). Professor do Curso de Graduação em Enfermagem da Universidade Veiga de Almeida (UVA). ORCID: 0000-0003-3213-5527

\section{Milena Preissler das Neves}

Administradora. MBA em Marketing pelo Centro Universitário Ritter dos Reis (UniRitter). Acadêmica Enfermagem da Universidade Veiga de Almeida, membro e fundadora da Liga acadêmica de Saúde Coletiva da Universidade Veiga de Almeida (LASC - UVA) e membro da Liga Acadêmica de Enfermagem em Terapia intensiva e emergência (LAETIE - UNIRIO).

ORCID: 0000-0002-3890-924X

\section{Roni Robson da Silva}

Acadêmico de enfermagem da Universidade Veiga de Almeida (UVA).

ORCID: 0000-0001-6010-6438

\section{İcaro Ferracini de Alencar}

Acadêmico de Medicina da Universidade Iguaçu.

ORCID: 0000-0003-3852-7767

\section{Michael da Silva de Araújo}

Engenheiro de Produção e Administrador. Especialista pela Universidade Federal de Itajubá. Docente da FAETEC/RJ.

ORCID: 0000-0002-5389-5712

\section{INTRODUÇÃO}

0 uso do álcool marca a relação entre os seres humanos, estando presente em diferentes contextos, com objetivos e motivações e formas diversas. O consumo de álcool vem desde a antiguidade, e o chamado alcoolismo foi tratado de acordo com a história por várias formas de exclusão, passando a ser alvo de pesquisa e de inúmeras explicações e caracterizações manifestadas das mais diversas formas. A história demonstrou que a pessoa com problemas de uso abusivo de álcool e outras substâncias recebe olhares voltados mais para a punição do que ao tratamento e reinserção. $\mathrm{O}$ uso abusivo destas substâncias gera conflitos e desestrutura o indivíduo no convívio familiar, social e profissional, acarretando problemas de ordem emocional, física e social. ${ }^{(1)}$

O relatório Mundial sobre Drogas 2020, publicado pelo Escritório das Nações Unidas sobre Drogas e Crime (UNODC) revelou que cerca de 269 milhóes de pessoas usaram drogas no mundo no ano 2018, se comparado ao ano de 2009, houve um au- mento de 30\%. O estudo revelou que pessoas em situação de vulnerabilidade com a pandemia do COVID-19 aumentaram o consumo de entorpecentes, tornando-as mais vulneráveis ao uso, ao tráfico e ao cultivo de drogas para obterem sustento. ${ }^{(2)}$

O referido relatório destacou que o uso da cannabis é a droga mais utilizada no mundo. Quanto ao álcool, o Relatório Global sobre Saúde e Álcool de 2018, elaborado pela Organização Mundial da Saúde (OMS), destacou que ele estaria associado a cerca de $18 \%$ dos casos de violência doméstica. Com o isolamento imposto pela COVID-19 especialmente as medidas de distanciamento social, que implicam em uma maior permanência em casa, têm favorecido o uso abusivo de álcool e facilitado atos violentos. ${ }^{(3)}$

Historicamente o uso de substâncias psicoativas, principalmente o álcool, têm sido uma constante na vida do ser humano. Na literatura, seu uso é descrito com várias finalidades, tais como: rituais, eventos comemorativos, reuniões, cultos sagrados e profanos, para alívio das dores, busca de prazer e como meio para atingir estados até então não alcançados, denotando também desregramento, contestação, ameaça, poder, desqualificação. ${ }^{(4)}$

De acordo com a OMS o uso nocivo de álcool está relacionado a 3 milhões de mortes, representando 5,3\% de todas as mortes. Para além disto, este uso nocivo é um fator causal para mais de 200 doenças e lesões. O consumo de álcool causa mortes e incapacidades precoces. $\mathrm{Na}$ faixa etária de $20 \mathrm{a}$ 39 anos, aproximadamente $13,5 \%$ do total de mortes são atribuíveis ao álcool. Além da incidência de doenças infecciosas, tais como tuberculose e HIV/aids. ${ }^{(3)}$

Entre os efeitos causados pelo consumo de bebidas alcoólicas, destacam-se o aumento do risco para problemas sociais, de trabalho, familiares, físicos e legais, o que o configura como problema de saúde pública. Observa-se crescimento do consumo de álcool paralelamente ao aumento dos problemas sociais, associado ao estigma e ao preconceito sofrido pelos alcoolistas, que são vistos, na maioria das vezes, como pessoas indesejáveis, inconvenientes, desmoralizadas e indisciplinadas. ${ }^{(5)}$

$\mathrm{Na}$ área da saúde, os enfermeiros são os que mantem contato maior com os usuá- 
rios dos serviços de saúde, tendo assim, um grande potencial para reconhecer os problemas relacionados ao uso do álcool e desenvolver ações assistenciais. A atuação dos enfermeiros na área de adições é muito importante. Por esta razão, é importante que o profissional tenha conhecimentos, sobre o consumo de drogas lícitas como o álcool, e sua prevalência, para que possa atuar com eficiência junto as pessoas em situação de dependência química. ${ }^{(6)}$

Estudos epidemiológicos em âmbito internacional e nacional tem apontado o crescimento do número de mulheres consumidoras de álcool. Segundo eles, este quantitativo triplicou nos últimos anos. Considera-se uso abusivo de álcool a ingesta de quatro ou mais doses para mulheres, $\mathrm{e}$ cinco ou mais para homens em uma mesma ocasião. Estima-se que duas, em cada cinco pessoas da população mundial, fazem consumo de álcool, destas 20\% fazem nocivamente. $\mathrm{O}$ consumo pode trazer vários prejuízos de ordem psíquica, espiritual, física ou social para o indivíduo e a coletividade, é um fenômeno que constitui um dos maiores e mais onerosos problemas de saúde pública ao redor do mundo. ${ }^{(7)}$

Para além dos danos à saúde as consequências do consumo de álcool atingem familiares, vizinhos, colegas e demais pessoas de seu convívio social, possíveis vítimas do consumo de outra pessoa e não do próprio. ${ }^{(8)}$ No entanto, a OMS aponta alguns avanços em âmbito mundial alcançados pelas políticas de Redução do Uso Abusivo do Álcool adotadas em 194 países. ${ }^{(3)}$

Um outro problema relacionado em algumas situações ao consumo abusivo de álcool relaciona-se a redução da ansiedade, bem como à desinibição, está associada a crença de que o consumo aumentaria o prazer sexual, faz com que bebidas alcoólicas sejam facilmente consumidas antes ou durante os atos sexuais. Constituindo-se um fator de risco para infecções sexualmente transmissíveis, visto que pessoas que consomem bebidas alcoólicas em contextos nos quais praticam sexo podem tender a não utilizar preservativo nos atos sexuais, a trocar de parceiros com mais frequência, a ter parceiros casuais e a até mesmo praticar sexo grupal. ${ }^{(9)}$
$\mathrm{O}$ inquérito denominado vigitel realizado através de contatos telefônicos pelo Ministério da Saúde revelou que nas 27 capitais brasileiras pesquisadas a frequência de consumo abusivo de álcool nos 30 dias anteriores à pesquisa foi de 18,8\%. Entre as mulheres a frequência de consumo abusivo aumentou de 11\% em 2018 para 13,3\% em 2019. Em ambos os sexos, a frequência de consumo tendeu a reduzir com a idade e a aumentar com os níveis de escolaridade. Com relação ao hábito de beber e dirigir, $5,6 \%$ dos entrevistados relataram esse comportamento. ${ }^{(10)}$

\section{Para além dos}

danos à saúde as

consequências do

consumo de álcool

atingem familiares,

vizinhos, colegas e

demais pessoas de

seu convívio social,

possíveis vítimas do

consumo de outra

pessoa e não do

próprio.

É imprescindível considerar a atuação dos enfermeiros juntos às adições, principalmente no que diz respeito as ações que desenvolvem junto a pessoas com problemas relacionados ao uso abusivo de álcool. Desta forma, este estudo tem como objetivo descrever o estado da arte da atuação de enfermagem junto a pessoas com problemas de uso abusivo de álcool, expresso na literatura científica nacional.

\section{MÉTODO}

Trata-se de uma revisão integrativa de literatura, que analisou e sintetizou os achados de publicações, produzidas por enfermeiros, que abordam os cuidados com pessoas com problemas pelo uso abusivo do álcool.

$\mathrm{Na}$ revisão integrativa, foram desenvolvidas as seis etapas que a compõem, com a seguinte questão norteadora: "Qual a percepção dos enfermeiros para identificar o usuário de drogas lícitas, como o álcool expresso na literatura científica"? Através do fluxograma PRISMA, dividido em quatro etapas (Figura 1).

Foram respeitadas as 6 fases da RIL, a saber: a primeira compreendeu a elaboração da questão norteadora "O que os enfermeiros brasileiros publicaram sobre uso abuso de álcool?". Na segunda fase buscou-se a amostragem na literatura, através de artigos nas bases de dados Literatura Latino-Americana e do Caribe em Ciências da Saúde (LILA$\mathrm{CS}$ ), Base de Dados de Enfermagem (BDENF) e Scientific Eletronic Library Online (SciELO). Na terceira fase, compreendeu-se a coleta de dados utilizando os seguintes descritores combinados: Enfermagem and Alcoolismo, e a expressão "uso abusivo de álcool" em periódicos de enfermagem. Os critérios de inclusão definidos para a seleção dos artigos foram: artigos publicados em português; na íntegra; com recorte temporal de 2012 à 2020. Após leitura exaustiva dos artigos, foram excluídos os que não guardavam relação com a temática estudada e/ ou que não atendiam aos critérios de inclusão anteriormente descritos. A quarta fase compreendeu a análise crítica dos estudos selecionados, em busca de evidências científicas e sistemas de classificações, adotou-se as sugeridas por Oxford Centre for Evidence- Based Medicine. Assim como o método de Melnyk e Fineout-Overholt. ${ }^{(11)}$ 
$\mathrm{Na}$ quinta fase foi feita a discussão dos resultados, compreendendo a interpretação e síntese dos resultados, assim como a comparação evidenciados, para melhor compreensão dos dados foram criadas categorias temáticas. E a última e sexta fase, foi a apresentação da revisão integrativa.

\section{RESULTADOS}

Os resultados evidenciaram o perfil das produções acadêmicas produzidas por enfermeiros sobre o uso abusivo do álcool. Destacaram os aspectos relacionados ao cuidado com esta clientela, bem como impasses na assistência. Para melhor compreensão dos

Figura 1 - Síntese dos resultados da revisão sistemática. Rio de Janeiro, RJ, 2021.

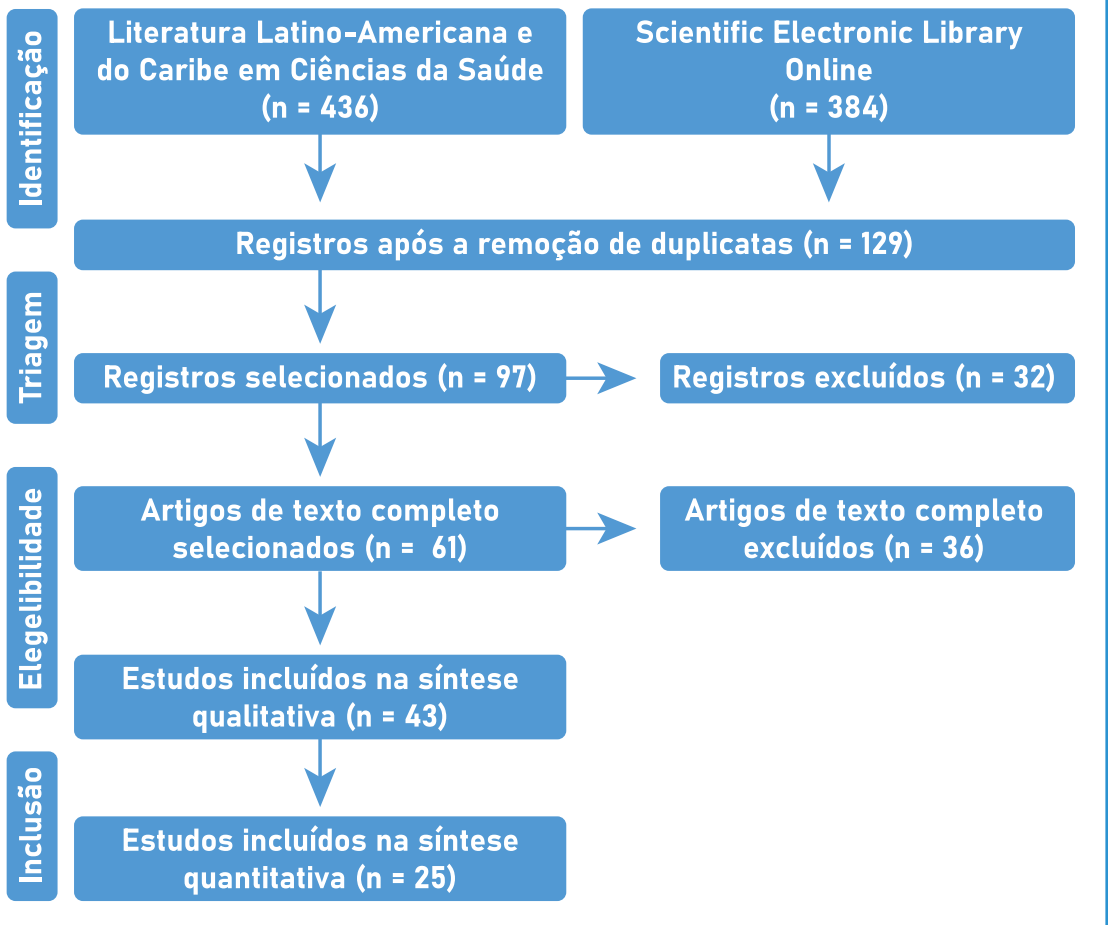

Fonte: Elaborado pelos autores, 2021

dados, optou-se por construir 2 categorias temáticas, denominadas de Atenção básica e sua relação com a saúde mental; e Atenção hospitalar em saúde mental, ambas possuem subcategorias que estão explicitadas na tabela 1 abaixo. Sendo inclusive adotado o método de Melnyk e Fineout-Overholt, que objetiva a apreciação do nível de evidência.

$\mathrm{Na}$ categoria Atenção básica em saúde mental foram encontrados onze artigos produzidos por enfermeiros, que por sua vez foram divididos em subcategorias. A denominada Centros de atenção psicossocial, foram identificados quatro artigos. Dois artigos relatam sobre a atuação do CAPSad em assistência as pessoas com quadro de dependência química. Os achados revelaram que estes profissionais são considerados fundamentais no processo da transformação social, participando na implantação de programas e projetos de promoção da saúde, prevenção do uso e abuso de álcool e outras drogas, além da interação social.

Na subcategoria Estratégia de saúde da família foram identificados sete estudos. Destes três tiveram abordagem diferentes em relação ao atendimento da Rede de saúde ao usuário de álcool. Em outros dois, foi descrito a dificuldade que os enfermeiros têm ao proporcionar atendimento da rede de saúde, a falta de capacitação e de motivação da equipe de saúde é um dos desafios encontrados para atender esses usuários. Destaca-se que as equipes revelaram ter dificuldades em li-

Tabela 1 - Síntese dos estudos publicados por enfermeiros, Rio de Janeiro, RJ, 2021.

\begin{tabular}{|c|c|c|c|c|}
\hline CATEGORIA & SUBCATEGORIA & $\begin{array}{l}\text { QUANTIDADE } \\
\text { DE ESTUDOS }\end{array}$ & $\begin{array}{l}\text { NIVELL DE } \\
\text { EVIDÊNCIA }\end{array}$ & SÍNTESE \\
\hline \multirow{2}{*}{$\begin{array}{l}\text { Atenção básica e sua } \\
\text { relação com a saúde } \\
\text { mental. }\end{array}$} & $\begin{array}{l}\text { Centros de atenção } \\
\text { psicossocial }\end{array}$ & 04 & V & $\begin{array}{l}\text { Os estudos destacaram a atuação dos enfermeiros } \\
\text { em CAPSad para a promoção da saúde e ressignifica- } \\
\text { ção de comportamentos. }\end{array}$ \\
\hline & $\begin{array}{c}\text { Estratégia de saúde } \\
\text { da familia }\end{array}$ & 07 & V & $\begin{array}{l}\text { As pesquisas destacaram a atuação dos enfermeiros } \\
\text { com pessoas com problemas relacionados ao uso } \\
\text { abusivo de álcool na atenção básica. }\end{array}$ \\
\hline \multirow{2}{*}{$\begin{array}{l}\text { Atenção hospitalar em } \\
\text { saúde mental }\end{array}$} & Hospitais gerais & 08 & v & $\begin{array}{l}\text { Abordou-se a percepção dos profissionais de en- } \\
\text { fermagem em casos de emergências psiquiátricas } \\
\text { envolvendo o uso abusivo de álcool. }\end{array}$ \\
\hline & $\begin{array}{l}\text { Hospitais } \\
\text { psiquiátricos }\end{array}$ & 06 & V & $\begin{array}{l}\text { Os estudos destacaram os efeitos deletérios da inter- } \\
\text { nação psiquiátrica e do estigma gerados em pessoas } \\
\text { com problemas com uso abusivo de álcool. }\end{array}$ \\
\hline
\end{tabular}




\section{artigo}

dar com essa clientela, assim como sentem-se desmotivados por vezes com as recaídas que esse tipo de usuário apresenta.

Quanto à categoria Atenção hospitalar em saúde mental, emergiram as subcategorias Hospitais gerais; e Hospitais psiquiátricos. $\mathrm{Na}$ primeira um estudo abordou a segurança do paciente em serviços de urgência, especificamente dos usuários de bebida alcoólica e, uma proposta de padrões mínimos de assistência de enfermagem necessários à atenção segura no atendimento inicial aos usuários foi proposta. Em relação à segurança dos pacientes, os resultados mostraram que a qualidade na assistência à saúde e é uma preocupação do Sistema Único de Saúde (SUS).

Ainda nesta subcategoria, um estudo identificou duas temáticas relacionadas com a percepção dos profissionais de Enfermagem sobre o cuidado destinado a pessoas com problemas relacionados ao uso abusivo de álcool: a percepção sobre o cuidado de Enfermagem para está clientela e o e a cronicidade que pode ser gerada com o uso abusivo.

Em relação a subcategoria Hospital psiquiátrico os estudos abordaram o perfil dos pacientes internados, destacando-se que apesar do advento da reforma psiquiátrica ainda foram internados nestas instituições com características asilares. Destacaram-se os estudos que apontaram uma prevalência de mulheres internadas por questões associadas ao uso abusivo de álcool. Predominando as adultas, solteiras e com baixo nível de escolaridade. Bem como destacava os clientes que após a alta hospitalar, eram acompanhados por serviços especializados, e ainda assim, apresentavam recaídas.

\section{DISCUSSÃO}

Referente à categoria Atenção básica e sua relação com a saúde mental, as subcategorias analisadas apresentaram uma visão clara sobre $\mathrm{o}$ atendimento especializado, voltado as pessoas com quadro de dependência química. Todos os artigos desta categoria analisados demonstram a necessidade de atualização dos profissionais de enfermagem, assim como a exigência de habilidades nos atendimentos destinados a essa clientela.

Ao longo das últimas décadas, várias trans- formações no modelo de atenção em saúde mental, que priorizam ações voltadas para a inclusão social, cidadania e autonomia das pessoas portadoras de transtornos mentais. No que tange as pessoas que fazem uso abusivo de álcool, há um cuidado de não estigmatizar e nem institucionalizar estes sujeitos. Inclusive pelo fato desta droga ser socialmente aceita por ser lícita, contribuindo assim para um aumento de seu consumo. ${ }^{(12)}$ Destaca-se, que a continuidade dos cuidados é um elemento central de uma atenção primária efetiva; e quando há um relacionamento contínuo entre os profissionais de saúde e os pacientes, a qualidade dos serviços de saúde mental na atenção primária tende a aumentar. ${ }^{(13)}$

Neste sentido os centros de atenção psicossocial têm papel fundamental, inclusive o Global Mental Health (GMH) é um campo de estudos e de práticas que objetivam reduzir as iniquidades no acesso e nos cuidados à saúde mental em escala global. Entretanto, o abuso de substâncias aumentou 38\% e representam 7,4\% da carga mundial total de problemas de saúde. ${ }^{(13)}$

A subcategoria Estratégia de saúde da família verificou-se que o atendimento oferecido a pessoa com problema abusivo com o álcool no contexto da comunidade, em alguns momentos é repleto de estigmas e preconceitos. É importante identificar o padrão de consumo, bem como sensibilizar a comunidade para a percepção dos danos à saúde que o uso abusivo pode causar. As ações primárias de saúde devem estar focadas na detecção precoce, inclusive existem instrumentos para rastreamento, assim como lançar mão de intervenções breve, de técnicas de tratamento baseados em aspectos cognitivos comportamentais. ${ }^{(14)}$

Na categoria Atenção hospitalar em saúde mental, na leitura da subcategoria hospital emergência, a segurança do paciente, quanto ao atendimento a ser recebido em uma sala de emergência, deve ter uma proposta mínima de assistência de enfermagem necessários à atenção segura no atendimento inicial aos usuários de bebida alcoólica. ${ }^{(11)}$

Sobre está temática pouco se escreve em saúde mental, é preciso considerar um manejo diferenciado, haja vista que o público atendido pode predispor à ocorrência de alguns eventos não desejados, danosos ao paciente, tais como autoagressão, violência e suicídio. ${ }^{(15)}$

Em relação a percepção do enfermeiro, verificaram que o cuidado é limitado apenas aos procedimentos técnicos, e o não seguimento das normas da instituição atrapalha a prestação desse cuidado; enquanto o alcoolismo é percebido como uma doença crônica, que envolve fatores biopsicossociais, sem cura, mas que possui tratamento. Em sua maioria, os profissionais da equipe de Enfermagem reconhecem o alcoolismo como doença crônica. ${ }^{(2)}$

Quanto a subcategoria, hospitais psiquiátricos, os estudos constantes revelaram que o perfil dos pacientes assistidos demonstrou uma certa prevalência de mulheres que faziam uso abusivo de álcool, a predominância de adictas adultas com média de idade foi de 41,4 anos. Observou-se também, baixa escolaridade dos participantes, indicando que tantos homens e mulheres tem um perfil similar neste sentido. Os estudos apontaram que os sujeitos do estudo reconhecem os prejuízos sociais, assim como uma queda de desempenho no trabalho em decorrência do uso abusivo do álcool. ${ }^{(12)}$

Destaca-se que graças ao advento da reforma psiquiátrica e das legislações destinadas a clientela portadora de sofrimento psíquico, ao longo dos anos observa-se um progressivo declínio de internações, bem como redução de leitos e instituições para essa finalidade. Para além disto, observa-se um aumento no empoderamento dos clientes, tornando-os protagonista do processo de cuidar e não meros sujeitos como outrora. ${ }^{(16)}$

\section{CONCLUSÃO}

Para além de possíveis conclusões, o álcool é um grave problema de saúde pública mundial, por ser lícito permeia historicamente diferentes momentos e ritos de passagem da vida humana. Associado a outras substâncias psicoativas ele pode ser potencializado, colocando o sujeito em uma situação de maior vulnerabilidade. Em diferentes contextos profissionais de enfermagem contribuem no tratamento e no processo de cuidar destes sujeitos.

Através deste estudo foi possível traçar um panorama das produções acadêmicas de en- 
fermagem, as quais destacassem a atuação do profissional enfermeiro. No entanto, este estudo apresentou limitações. A principal referente a escassez de pesquisas que abordassem essa temática. Sugere-se assim, a realização de novos e mais complexos estudos que possam principalmente descrever possíveis impactos que o consumo abusivo de álcool pode ter trazido durante a pandemia de COVID-19.

Por ser uma temática controversa em al- guns momentos pela legalidade desta substância, estudos como este serão sempre pertinentes para uma melhor compreensão deste fenômeno, bem como para a instrumentalização do cuidar em enfermagem.

\section{REFERÊNCIAS}

1. Barbosa I de SF, Mendonça C de F de S, Silva RR da, Silva LA, Silva MVG da, Neves MP das, Souza MVL de, Alencar Ícaro F de, Fontes TV, Ignácio LP, Oliveira JVE, Souza M das GG de, Paulino E de FR, Araujo MS, Souza DAC. Cuidados destinados a pessoas com problema relacionado ao uso abusivo de álcool: um estudo de revisão integrativa de literatura. RSD [Internet]. $24^{\circ}$ de novembro de 2020 [citado $17^{\circ}$ de janeiro de 2021];9(11):e52091110062. Disponivel em: https:/rsdjournal.org/index.php/rsd/article/view/10062

2. United Nations. Unodc. World Drug Report 2020. [Internet]. June 2020. 2020 [cited 2021 Jan 16] Available from: https:/wdr.unodc.org/ wdr2020/field/WDR20_Booklet_2.pdf

3. World Health Organization. Global status report on alcohol and health 2018. [Internet]. [cited 2021 Jan 17] . Available from: https:/apps.who. int/iris/rest/bitstreams/1151838/retrieve

4. Silva AP da, Perrelli JGA, Guimarães FJ, Mangueira S de O, Cruz SL, Frazão I da S. Identificação do diagnóstico de enfermagem autocontrole ineficaz da saúde em alcoolistas: um estudo descritivo. Rev. Eletr. Enferm. [Internet]. $31^{\circ}$ de dezembro de 2013 [citado $17^{\circ}$ de janeiro de 2021];15(4):932-9. Disponivel em: https:/revistas.ufg.br/fen/article/ view/19841

5. Moll, MF, Ventura, CAA, Pires, FC, Boff, NN, Silva, CBF, Oliveira, PC. (2019). Síndrome de abstinência alcoólica: conhecimentos e cuidados da Enfermagem na clínica cirúrgica do hospital geral. SMAD. Revista eletrônica saúde mental álcool e drogas, 15(3), 1-8. https:/dx.doi. org/10.11606/issn.1806-6976.smad.2019.150193

6. Robson SR, Preissler NM, Andrade SL, Godoy SMV, Leite HR, Bertolossi MC. Consumo de drogas psicoativas em contexto sexual entre homens gays como fator de risco para transmissão de HIV/Aids. Glob Acad Nurs [Internet]. $31^{\circ}$ de dezembro de 2020 [citado $17^{\circ}$ de janeiro de 2021];1(3):e57. Disponível em: http:/globalacademicnursing.com/index. php/globacadnurs/article/view/98

7. Silva MGB, Lyra TM, Diniz GT. O padrão de consumo de álcool entre as usuárias das Unidades de Saúde da Familia no município do Recife (PE). Saúde em Debate [online]. v. 43, n. 122 [Acessado 17 Janeiro 2021] , pp. 836-847. Disponível em: <https:/doi.org/10.1590/01031104201912214>. ISSN 2358-2898. https:/doi.org/10.1590/01031104201912214.

8. Monteiro MG. Políticas públicas para a prevenção dos danos relacionados ao consumo de álcool. Epidemiol. Serv. Saúde [Internet]. $2016 \mathrm{Mar}$ [cited 2021 Jan 17]; 25(1):171-174. Availablefrom:http:/www.scielo.br/ scielo.php?script=sci_arttext\&pid=S2237-96222016000100171\&lng=en. https:/doi.org/10.5123/s1679-49742016000100017.

9. Cardoso LRD, Malbergier A, Figueiredo TFB. O consumo de álcool como fator de risco para a transmissão das DSTs/HIV/Aids. Rev. psiquiatr. clín. [Internet]. 2008 [cited 2021 Jan 17] ; 35( Suppl 1 ): 70-75. Available from: http:/www.scielo.br/scielo.php?script=sci_arttext\&pid=S0101-60832008000700015\&lng=en. https:/doi.

\section{org/10.1590/S0101-60832008000700015.}

10. Brasil. Ministério da Saúde. Secretaria de Vigilância em Saúde. Departamento de Análise em Saúde e Vigilância de Doenças Não Transmissíveis. Vigitel Brasil 2019 : vigilância de fatores de risco e proteção para doenças crônicas por inquérito telefônico : estimativas sobre frequência e distribuição sociodemográfica de fatores de risco e proteção para doenças crônicas nas capitais dos 26 estados brasileiros e no Distrito Federal em 2019 [recurso eletrônico] / Ministério da Saúde, Secretaria de Vigilância em Saúde, Departamento de Análise em Saúde e Vigilância de Doenças não Transmissíveis. - Brasilia: Ministério da Saúde, 2020.

11. Amaral RT, Soares R FS, Silva SMTC, Neves MP, Silva LA, Silva RR. Identificação e análise de Garbage code ocorridos no preenchimento da Declaração de Óbito no Hospital Geral de Nova Iguaçu - RJ, uma realidade a ser modificada. RSD [Internet]. $7^{\circ}$ de dezembro de 2020 [citado $17^{\circ}$ de janeiro de 2021];9(11):e91591110658. Disponivel em: https:/rsdjournal. org/index.php/rsd/article/view/10658

12. Correia VR, Barros S, Colvero LA. Saúde mental na atenção básica: prática da equipe de saúde da familia. Rev. esc. enferm. USP [Internet]. 2011 Dec [cited 2021 Jan 17] ; 45( 6 ): 15011506. Available from: http:/www.scielo.br/scielo.php?script=sci_arttext\&pid=S0080-62342011000600032\&lng=en. https:/doi. org/10.1590/S0080-62342011000600032.

13. Wenceslau LD, Ortega F. Saúde mental na atenção primária e Saúde Mental Global: perspectivas internacionais e cenário brasileiro. Interface (Botucatu) [Internet]. 2015 Dec [cited 2021 Jan 17] ; 19( 55 ): 11211132. Available from: http:/www.scielo.br/scielo.php?script=sci_arttext\&pid=S1414-32832015000401121\&Ing=en. Epub Aug 21, 2015. https:/doi.org/10.1590/1807-57622014.1152.

14. Soares J, Vargas D, Formigoni Maria Lucia Oliveira de Souza. Knowledge and attitudes of nurses towards alcohol and related problems: the impact of an educational intervention. Rev. esc. enferm. USP [Internet]. 2013 Oct [cited 2021 Jan 17] ; 47( 5 ): 11721179. Available from: http:/www.scielo.br/scielo.php?script=sci_arttext\&pid=S0080-62342013000501172\&Ing=en. https:/doi. org/10.1590/S0080-623420130000500023.

15. Vantil FCS, Lima EFA, Figueiredo KC, Massaroni L, Sousa Al, Primo CC. Segurança do paciente com transtorno mental: construção coletiva de estratégias. Rev. Bras. Enferm. [Internet]. 2020 [cited 2021 Jan 16] ; 73( 1 ): e20170905. Available from: http:/www.scielo.br/scielo. php?script=sci_arttext\&pid=S0034-71672020000100158\&lng=en. Epub Feb 10, 2020. http:/dx.doi.org/10.1590/0034-7167-2017-0905.

16. Bragé ÉG, Ribeiro LS, Rocha DG, Ramos DB, Vrech LR, LAJB. Perfil de internações psiquiátricas femininas: uma análise crítica. J. bras. psiquiatr. [Internet]. 2020 July [cited 2021 Jan 16] ; 69(3): 165-170. Available from: http:/www.scielo.br/scielo.php?script=sci_arttext\&pid=S0047-20852020000300165\&Ing=en. Epub June 24, 2020. https:/doi.org/10.1590/0047-2085000000275. 